\title{
Clostridium difficile presence in Spanish and Belgian hospitals
}

\author{
C. Rodriguez a, * , J. Fernandez ${ }^{\text {b }}$, J. Van Broeck ${ }^{\text {c }}$, B. Taminiau ${ }^{\text {a }}$, V. Avesani ${ }^{\text {c }}$, J.A. Boga ${ }^{\text {b }}$, \\ F. Vazquez ${ }^{\text {b }}$, M. Delmée ${ }^{c}$, G. Daube ${ }^{\text {a }}$ \\ a Food Science Department, FARAH, Faculty of Veterinary Medicine, University of Liège, Liège, Belgium \\ b Service of Microbiology, Hospital Universitario Central de Asturias, Oviedo, Spain \\ ${ }^{\mathrm{c}}$ Microbiology Unit, Faculty of Medicine, Catholic University of Louvain, Brussels, Belgium
}

\section{A R T I C L E I N F O}

\section{Article history:}

Received 13 July 2016

Received in revised form

1 September 2016

Accepted 6 September 2016

Available online 9 September 2016

\section{Keywords:}

C. difficile

Hospitals

Epidemiology

\begin{abstract}
A B S T R A C T
Clostridium difficile is recognised worldwide as the main cause of infectious bacterial antibioticassociated diarrhoea in hospitals and other healthcare settings. The aim of this study was to first survey $C$. difficile prevalence during the summer of 2014 at the Central University Hospital of Asturias (Spain). By typing the isolates obtained, it was then possible to compare the ribotype distribution at the Spanish hospital with results from the St Luc University Hospital in Belgium over the same period. The prevalence of positive cases reported in Spain and Belgium was $12.3 \%$ and $9.3 \%$ respectively. The main PCR-ribotypes previously described in Europe were found in both hospitals, including 078, 014, 012, 020 and 002. In the Spanish hospital, most of the C. difficile-positive samples were referred from oncology, acute care and general medicine services. In the Belgian hospital the majority of positive samples were referred from the paediatric service. However, a high percentage of isolates from this service were nontoxigenic. This study finds that the presence and detection of $C$. difficile in paediatric and oncology services requires further investigation.
\end{abstract}

() 2016 Elsevier Ltd. All rights reserved.

\section{Introduction}

Clostridium difficile is currently one of the most largely studied pathogenic bacteria in the world and is considered as the major cause of nosocomial antibiotic-associated diarrhoea and colitis in industrialised countries [1]. Clinical manifestations of $C$. difficile infection (CDI) range from mild or moderate diarrhoea to fulminant and sometimes fatal pseudomembranous colitis [2]. Normally, the diarrhoea has been described to appear $48-72 \mathrm{~h}$ post infection and characterised as non-haemorrhagic and watery, accompanied with abdominal pain, fever and leucocytosis [3]. However, the worst outcomes are sepsis and death, which is observed in $17 \%$ of CDI cases [4]. The highest incidence and mortality rate is usually reported among patients of advanced age who have had a stay in a healthcare setting [5].

A recent review of $C D I$ cost-of-illness attributes a mean cost ranging from $\$ 8911$ to $\$ 30,049$ per hospitalised patient in the USA [6] and around $€ 3000$ million total per annum in Europe [7]. In addition, in many hospitals the diagnosis strategy remains

\footnotetext{
* Corresponding author.

E-mail address: c.rodriguez@ulg.ac.be (C. Rodriguez).
}

suboptimal and a proportion of infections may remain undiagnosed [8]. In the past decade, an increase in the incidence and severity of the infection has been reported in various healthcare settings among many countries [9]. This situation was attributed to the emergence of a new epidemic and hypervirulent $C$. difficile strain, identified as PCR-ribotype 027 (NAP1 or North American pulsed field type 1) [10]. Since 2003, in the United States and Canada, studies have shown an increase in the number and severity of CDI cases, including an increase in the case fatality, mortality and colectomy rates [11]. The situation presented by studies in North America is mirrored in Europe. In 2008, the PCR-ribotype 027 was detected in 16 European countries and caused outbreaks in Belgium, Germany, Finland, France, Ireland, The Netherlands, Switzerland and the United Kingdom [11,12]. However, in a further epidemiology study conducted in Europe, the most prevalent PCRribotypes were identified as 014/020 (15\%), 001 (10\%) and 078 (8\%), while PCR-ribotype 027 was less prevalent (5\%) [12]. Surveillance data for Belgium from 2008 to 2010 showed a stable incidence of CDI in Belgian hospitals, and even a decrease in 2010. In addition, PCR-ribotype 027 was the most prevalent type during the years 2007-2009 [13]. A further study reporting CDI ribotype distribution in Belgian hospitals between 2008 and 2010 described a decrease in cases caused by PCR-ribotype 027 (from 55\% in 2008 to 
$28 \%$ in 2010). In contrast, the proportion of other PCR-ribotypes involved in CDI increased, such as ribotype 014 (from $20 \%$ in 2008 to $33 \%$ in 2010) and ribotype 078 (from $11 \%$ in 2009 to $23 \%$ in 2010) [14]. Meanwhile, a prospective study conducted in 2009 in the region of Barcelona (Spain) identified the main PCR-ribotypes associated with CDI as 241 (26\%), 126 (18\%), 078 (7\%) and 020 (5\%), while PCR-ribotype 027 was not detected [15]. In a later study conducted in the region of Madrid (Spain) from January to June 2013, most of the isolates associated with a CDI case possessed binary toxin and were classified as PCR-ribotype 078/126 (90.7\%) [16]. Consistent with these reports, Weber et al. [17] studied C. difficile clinical isolates recovered at the reference hospital of the Balearic Islands (Spain) between August 2007 and April 2011. The authors detected a total 43 different PCR-ribotypes with a higher prevalence of types 014 (34\%), 078 (13\%) and 001 (5\%). As in other Spanish studies, none of the isolates were identified as PCRribotype 027.

The aim of this study was to survey the $C$. difficile circulation during the summer of 2014 at the Central University Hospital of Asturias (Spain), a provincial hospital located in the North of Spain. By typing of all the isolates obtained, it was then possible to compare the ribotype distribution at the Spanish hospital with results from the St Luc University Hospital in Belgium over the same period.

\section{Methods}

\subsection{Hospital selection, data and sampling}

The Central University Hospital of Asturias (HUCA) located in Oviedo (Asturias, Spain), is the referral hospital of the Health Service of the Principality of Asturias. Overall, the hospital has 17 buildings with a total of 1324 beds, 29 operating rooms, 203 consultation rooms (for outpatients) and 123 emergency rooms.

During the 4-month period from July to October 2014, all samples from outpatients and hospitalised patients suspected of being infected with $C$. difficile were tested. Stool consistency of samples was evaluated using the Bristol Stool Chart (BSC). Samples were documented for data relating to clinical history, diagnosis and treatment received, including the prescription of antimicrobial agents. Numerical identification was used for all samples to guarantee patient anonymity.

\subsection{C. difficile rapid detection}

Initial screening for $C$. difficile presence was performed using a rapid membrane enzyme immunoassay for the simultaneous detection of $C$. difficile glutamate dehydrogenase antigen and toxins A and B (Cdiff QuickChek Complete ${ }^{\circledR}$ TechLab, Blaclsburg, USA). In the case of doubtful results or glutamate dehydrogenase antigen testing positive and toxins A and B testing negative, GenomEra CDX System C. difficile (Abacus Diagnostica, Turku, Finland) was performed for rapid identification of toxin B. These tests were applied only in semisolid, mushy stools and watery/entirely liquid faeces (Bristol stool chart levels 4 to 7) while samples outside this range were discarded. This analysis constituted the routine protocol followed in the hospital laboratory for the diagnosis of CDI. If various stool samples were received from the same patient, a second analysis was only performed if the first $C$. difficile screening was made at least one month prior.

\subsection{Culture, identification and characterisation}

All specimens received in the laboratory for $C$. difficile testing were cultured regardless of their classification in the BSC. Culture was carried out as described previously [18]. Briefly, approximately $0.1 \mathrm{~g}$ of faeces was spread directly on cycloserine cefoxitin fructose agar taurocholate medium (CCFAT), freshly prepared in the laboratory. Plates were incubated anaerobically for $48-72 \mathrm{~h}$ at $37{ }^{\circ} \mathrm{C}$. The anaerobic atmosphere in the jar was created using AnaeroGen $^{\mathrm{TM}}$ sachet (Oxoid, Dardilly, France) and checked using an anaerobic indicator BR0055B (Oxoid). An enrichment step was also performed. One gram of faeces was inoculated into $9 \mathrm{ml}$ of CCFT (cycloserine cefoxitin fructose taurocholate) broth and incubated anaerobically for $72 \mathrm{~h}$ at $37^{\circ} \mathrm{C}$. A $10 \mu$ laliquot of the enriched broth was spread on CCFT plates and incubated anaerobically at $37{ }^{\circ} \mathrm{C}$ for 48-72 h. One presumptive colony per plate was subcultured onto blood agar 5\% Sheep Blood (Biorad, Temse, Belgium) and checked using a C. difficile latex agglutination rapid test Kit DR 1107A (Oxoid). Detection of a species-specific internal fragment of the tpi gene, toxin A and B genes, and CDT ( $c d t A)$ was performed according to the multiplex PCR protocol [18]. Sterile water and PCR-ribotype 027 strain were used as negative and positive controls respectively. Further toxin profile characterisation, deletions in the regulator gene $t c d C$, and gyrA mutation (a gene associated with moxifloxacin resistance) were determined using the Genotype Cdiff system (Hain Lifescience, Nehren, Germany) according to the manufacturer's instructions. The supernatant from each pure culture was tested for cytotoxicity assay (TcdB) using confluent monolayer MRC-5 cells, as previously described [18].

All strains were ribotyped as described by Bidet et al. [19]. Amplicon sizes were analysed by capillary electrophoresis and profiles obtained were compared with those of reference strains from the European collection (Cardiff International number, Brazier classification) and with our own database (nomenclature beginning with UCL).

\subsection{Antibiotic resistance}

Susceptibility of the isolates to metronidazole, moxifloxacin and tetracycline was determined by Etest strips (Lucron ELITech Group, Zottegem, Belgium) on Brucella Blood Agar with hemin and vitamin K1 (Becton-Dickinson Benelux NV, Erembodegem, BE) according to the manufacturer's instructions. Plates were anaerobically incubated at $37{ }^{\circ} \mathrm{C}$ for $48 \mathrm{~h}$. The resistance ( $\mathrm{r}$ ) breakpoints for metronidazole (Met $\mathrm{r} \geq 32 \mu \mathrm{g} / \mathrm{ml}$ ), moxifloxacin (Mox $\mathrm{r} \geq 8 \mu \mathrm{g} / \mathrm{ml}$ ) and tetracycline (Tet $r \geq 8 \mu \mathrm{g} / \mathrm{ml}$ ) were those recommended by the Clinical and Laboratory Standard Institute (CLSI) [20]. Bacteroides fragilis ATCL was included as a quality control.

\subsection{Surveillance data in Belgium}

During the same study period (from July to October 2014) analysis of $C$. difficile ribotype distribution was made at the St Luc University Hospital (Brussels, Belgium) in order to compare PCRribotypes with those obtained in Spain. The Belgian hospital is an academic acute care hospital with a total of 1000 beds and the National Reference Center for C. difficile in humans in Belgium. All stools received in the laboratory were tested for the presence of C. difficile. Multiple stool samples from the same patient were all tested. Initial screening was made using Cdiff QuickChek Complete $^{\circledR}$ (TechLab). Culture of positive samples was performed on CHROMagar $C$. difficile Colorex ${ }^{\mathrm{TM}}$ (CHROMagar, BioTrading, Keerbergen, $\mathrm{BE}$ ) in order to isolate the strain (without enrichment, planting faeces directly on agar). Plates were incubated anaerobically for $24 \mathrm{~h}$ at $35^{\circ} \mathrm{C}$. All cultures were read with a binocular stereomicroscope, with the light beam through the Petridish under a certain angle. Strains were ribotyped as described above. The toxin gene profile of the strains and PCR-ribotype distribution in the Belgian hospital were then compared with those found in the 
Spanish ward.

\section{Results}

\subsection{C. difficile detection and strain characterisation in HUCA, Spain}

During the four-month study period, a total of 249 samples were screened for $C$. difficile presence using both the rapid enzyme test and culture analysis. Twelve additional samples were only examined by culture because they were classified outside of the range established (between 4 and 7) on the BSC. The overall prevalence of C. difficile in the faecal microbiota of patients studied was $12.3 \%$ (32/ 261 ). Of these, $69 \%$ were from adults aged more than 65 years old. Only following clinical suspicion, and a positive result for toxins A and/or B by rapid-test detection (Cdiff QuickChek Complete ${ }^{\circledR}$ or GenomEra CDX System C. difficile), a patient was considered to suffer from infection. With this approach, a total of 22 patients were diagnosed with CDI.

Altogether, 7 of the 32 C. difficile-positive samples detected (22\%) were referred from the oncology unit. However, the medical services which sent the most samples for the screening of $C$. difficile during the study period were the acute care unit $(28 / 261 ; 10.7 \%)$ and general medicine service $(37 / 261 ; 14.2 \%)$ (Table 1$)$. From these two services, $C$. difficile was isolated from six and five patients respectively. Regarding the type of faeces, six patients (2.3\%) suspected of CDI presented bloody stools but all tested negative for the bacterium. Most of the positive patients had mushy, watery or liquid stools $(\mathrm{n}=24)$. However, two patients with formed stools were also colonised with toxigenic $C$. difficile strains (Table 1 ).

Using rapid detection, 22 isolates tested positive for $t c d B$ gene while 6 isolates were found to be non-toxigenic. Characterisation of colonies obtained after culture of samples showed 27 toxigenic isolates (presence of toxins A and B). Of these 27 toxigenic isolates, 6 had binary toxin genes. None of the isolates presented a single base deletion at position 117 in the regulator tcdC gene. Two isolates showed an 18 bp deletion and eight presented a 39 bp deletion in the regulator $t c d C$ gene. Twenty different PCR-ribotypes were detected. Only nine isolates had a ribotype profile associated with a Cardiff collection reference number $(002(n=3), 078(n=2), 012$, 070,023 and 020). The remaining isolates were associated with an internal nomenclature (UCL), with a total of 14 different PCRribotypes identified. The only non-toxigenic PCR-ribotype was associated with the ribotype UCL9. In addition, this ribotype was the only that presented three types of deletions in the regulator tcdC gene (117 bp, $39 \mathrm{bp}$ and $18 \mathrm{bp}$ deletions) (Table 2). The same results (the presence of $C$. difficile in the sample with the same PCRribotypes) were obtained with and without enrichment of faeces. None of the patients were identified as carriers of more than one PCR-ribotype.

None of the isolates showed resistance to metronidazole. For tetracycline, eight isolates were fully resistant: PCR-ribotype 078 (two isolates), PCR-ribotype UCL16b (one isolate), PCR-ribotype UCL5a (four isolates) and PCR-ribotype 36a (one isolate). Resistance for moxifloxacin was detected in all isolates of PCR-ribotypes 078, UCL5a and UCL9. All of these isolates (PCR-ribotype 078, UCL9 and UCL5) presented a mutation in gyrA gen (Table 2).

\subsection{C. difficile detection and strain characterisation in St Luc University Hospital, Belgium}

Between July 2014 and October 2014 a total of 880 stool specimens were analysed from patients of the St Luc University Hospital suspected of having CDI. The national prevalence for $C$. difficile reported from the Belgian Reference Center was 9.3\%. A total of 127 C. difficile-positive samples were obtained from 87 patients. Seventeen of these positive patients (19.5\%) were referred from the paediatric service (including eight from the paediatric haematology unit and four from the intensive neonatology unit). The other medical services with significant numbers of $C$. difficile-positive patients were general internal medicine $(n=9 ; 10.3 \%)$, consultation $(\mathrm{n}=8 ; 9.2 \%)$, pneumatology-gastroenterology $(\mathrm{n}=8 ; 9.2 \%)$, nephrology-neurology $(n=7 ; 8 \%)$, surgery $(n=5 ; 5.7 \%)$ and

Table 1

Clinical data comparison between $C$. difficile-colonised and non-colonised patients.

\begin{tabular}{|c|c|c|}
\hline & C. difficile-negative patients (\%) & C. difficile-positive patients (\%) \\
\hline Total (\%) & 229 of 261 (87.7) & 32 of $261(12.3)$ \\
\hline Mean age in years & 60.5 & 63,6 \\
\hline \multicolumn{3}{|l|}{ Sorted by age } \\
\hline$>65$ years & $137(60)$ & $22(68.8)$ \\
\hline $20-<65$ years & $76(33.2)$ & $7(22)$ \\
\hline$>10-20$ years & $7(3.1)$ & $3(9.4)$ \\
\hline$>3, \leq 10$ years & $4(1.7)$ & $0(0)$ \\
\hline$\leq 3$ years & $5(2.2)$ & $0(0)$ \\
\hline \multicolumn{3}{|l|}{ Sorted by gender } \\
\hline Male & $125(54.6)$ & $22(68.8)$ \\
\hline Female & $104(45.4)$ & $10(31.3)$ \\
\hline \multicolumn{3}{|l|}{ Sorted by service } \\
\hline Oncology & $4(1.7)$ & $7(22)$ \\
\hline Acute care unit & $22(9.6)$ & $6(18.8)$ \\
\hline General medicine & $32(14)$ & $5(15.6)$ \\
\hline Nephrology & $14(6.1)$ & $2(6.3)$ \\
\hline Digestive & $20(8.7)$ & $2(6.3)$ \\
\hline Haematology & $5(2.2)$ & $2(6.3)$ \\
\hline General emergencies & $11(4.8)$ & $2(6.3)$ \\
\hline Paediatric emergencies & $6(2.6)$ & $1(3.1)$ \\
\hline Surgery & $1(0.4)$ & $1(3.1)$ \\
\hline Urology & $2(0.9)$ & $1(3.1)$ \\
\hline Other & $112(48.9)$ & $3(9.4)$ \\
\hline \multicolumn{3}{|l|}{ Sorted by type of sample } \\
\hline Bloody stools & $6(2.3)$ & $0(0)$ \\
\hline Mushy, watery or entire liquid stools (Bristol Stool Chart 6-7) & $163(62.5)$ & $24(75)$ \\
\hline Smooth and soft stools (Bristol Stool Chart 4-5) & $82(31.4)$ & $6(18.8)$ \\
\hline Formed stools (Bristol Stool Chart 1-3) & $10(3.8)$ & $2(6.3)$ \\
\hline
\end{tabular}


Table 2

Detailed information on C. difficile isolates at the HUCA hospital (Spain), including molecular characterisation and antibiotic resistance of the isolates.

\begin{tabular}{|c|c|c|c|c|c|c|c|c|c|c|c|c|c|c|}
\hline $\begin{array}{l}\text { Isolate } \\
\text { number }\end{array}$ & $\begin{array}{l}\text { Rapid } \\
\text { detection } \\
\text { GDH }\end{array}$ & $\begin{array}{l}\text { Rapid } \\
\text { detection } \\
\text { toxin } \mathrm{B}^{\mathrm{C}}\end{array}$ & $\begin{array}{l}\text { Rapid } \\
\text { detection toxin } \\
\mathrm{B}^{\mathrm{d}}\end{array}$ & $\begin{array}{l}\text { Culture } \\
\text { detection }\end{array}$ & $\begin{array}{l}\text { PCR- } \\
\text { ribotype }\end{array}$ & $\begin{array}{r}\text { CE tcdA } \\
t c d B\end{array}$ & $\begin{array}{l}c d t A \\
c d t B\end{array}$ & $\begin{array}{l}t c d C^{\mathrm{a}} \\
\text { MUT117 }\end{array}$ & $\begin{array}{l}t c d C^{\mathrm{a}} \\
39 b p\end{array}$ & $\begin{array}{l}t c d C^{a} \\
18 b p\end{array}$ & $\begin{array}{l}\text { gyrA } \\
\text { MUT }\end{array}$ & Metronidazole & Moxifloxacin & Tetracycline \\
\hline 10404 & + & - & + & + & UCL23b & ++ & - & - & - & - & - & - & - & - \\
\hline 10405 & + & + & + & + & 002 & $+\quad+$ & - & - & - & - & - & - & - & - \\
\hline 10406 & - & - & NT & + & 078 & $+\quad+$ & + & - & + & - & Mut1A & - & + & + \\
\hline 10407 & + & + & - & + & UCL16b & $+\quad+$ & - & - & - & - & - & - & - & + \\
\hline 10408 & + & + & + & + & UCL16 & ++ & - & - & - & - & - & - & - & - \\
\hline 10409 & + & - & - & + & UCL9 & $-\quad-$ & - & + & + & + & Mut1A & - & + & - \\
\hline 10410 & + & - & + & + & 012 & ++ & - & - & - & - & - & - & - & - \\
\hline 10411 & + & + & + & + & 078 & $+\quad+$ & - & - & + & - & Mut1A & - & + & + \\
\hline 10412 & + & - & - & + & UCL16 & $+\quad+$ & - & - & - & - & - & - & - & - \\
\hline 10413 & + & + & + & + & $\mathrm{UCL}^{\circ} 6^{\circ}$ & $+\quad+$ & - & - & - & - & - & - & - & - \\
\hline 10414 & + & + & + & + & UCL55a & $+\quad+$ & - & - & - & - & - & - & - & - \\
\hline 10415 & + & + & + & + & 070 & $+\quad+$ & - & - & - & - & - & - & - & - \\
\hline 10419 & + & + & + & + & 014 & $+\quad+$ & - & - & - & - & - & - & - & - \\
\hline 10420 & + & + & + & + & 002 & $+\quad+$ & - & - & - & - & - & - & - & - \\
\hline 10421 & + & - & + & + & UCL489 & $+\quad+$ & - & - & - & - & - & - & - & - \\
\hline 10422 & + & + & + & + & UCL5a & $+\quad+$ & + & - & + & - & Mut1A & - & + & + \\
\hline 10423 & + & + & + & + & 002 & $+\quad+$ & - & - & - & - & - & - & - & - \\
\hline 10454 & + & + & + & + & 023 & $+\quad+$ & + & - & + & + & - & - & - & - \\
\hline 10425 & + & - & - & + & UCL5a & $+\quad+$ & + & - & + & - & Mut1A & - & + & + \\
\hline 10426 & + & - & + & + & UCL36a & $+\quad+$ & - & - & - & - & - & - & - & + \\
\hline 10427 & - & - & NT & + & UCL499 & $+\quad+$ & - & - & - & - & - & - & - & - \\
\hline 10428 & + & + & + & + & UCL16i & $+\quad+$ & - & - & - & - & - & - & - & - \\
\hline 10429 & + & + & + & + & UCL5a & $+\quad+$ & + & - & + & - & Mut1A & - & + & + \\
\hline 10430 & + & + & + & + & UCL108 & $+\quad+$ & - & - & - & - & - & - & - & - \\
\hline 10431 & - & - & NT & + & UCL5a & $+\quad+$ & + & - & + & - & Mut1A & - & + & + \\
\hline 10432 & + & - & + & + & 020 & $+\quad+$ & - & - & - & - & - & - & - & - \\
\hline 10433 & + & + & + & + & UCL483 & $+\quad+$ & - & - & - & - & - & - & - & - \\
\hline 10155 & - & - & NT & + & UCL283 & $+\quad+$ & - & - & - & - & - & - & - & - \\
\hline 10559 & + & - & + & - & - & -- & - & - & - & - & - & - & - & - \\
\hline 10497 & + & - & + & - & - & $-\quad-$ & - & - & - & - & - & - & - & - \\
\hline 10584 & + & - & - & - & - & -- & - & - & - & - & - & - & - & - \\
\hline 10287 & + & - & - & - & - & -- & - & - & - & - & - & - & - & - \\
\hline
\end{tabular}

MUT: mutation; CE: cytotoxicity assay using confluent monolayer MRC-5 cells; NT: not tested.

+ : Positive result.

-: Negative result.

a Presence of deletions in the regulator gene tcdC (118bp-39bp-17bp).

b Presence of mutation in the gyrA gene associated with moxifloxacin resistance.

c Cdiff QuickCheck Complete TechLab.

d GenomeEra CDX System C. difficile.

subacute geriatrics ( $\mathrm{n}=5 ; 5.7 \%)$. The oncology service referred three positive patients (3.4\%). Twenty-one patients were $C$. difficilepositive in more than one sampling. The mean age of positive patients was 45 years old. However, 19 positive patients were children less than 10 years old, with a mean age of 1 year and 6 months in this group, and 9 of these patients were less than 1 year old. If the paediatric group is analysed separately, the mean age of positive adult patients was 60 years old (Table 3 ).

Eighty-three isolates (65\%) were positive for toxigenic culture and toxins A and B. Forty-four isolates were identified as nontoxigenic (Table 3). Overall, 37 different PCR-ribotypes were detected. Eight of these had ribotype profiles associated with the Cardiff collection under reference numbers $015(\mathrm{n}=1), 078$ $(\mathrm{n}=14), 106(\mathrm{n}=8), 014(\mathrm{n}=5), 020(\mathrm{n}=9), 056(\mathrm{n}=13), 012$ $(n=6)$ and $002(n=4)$. The remaining isolates were associated with an internal nomenclature (UCL), with a total of 29 different PCR-ribotypes identified, including all the non-toxigenic isolates (PCR-ribotypes UCL 36, UCL 9, UCL 110, UCL 122, UCL 257, UCL 384, UCL 46d) (Table 3).

\section{Discussion}

C. difficile continues to be the most common cause of healthcareassociated infection in the developed world. A previous European C. difficile infection hospital-based survey has shown that the incidence of CDI and the distribution of causative PCR-ribotypes differed greatly between hospitals [21]. In Spain, the number of toxin-positive cases reported varied between 5.5\% and 5.6\% (2008) [22], 9\% (2008) [21] and 6.0\%-6.5\% (2013) [22]. In this study the prevalence was higher than has been previously found in Spain. The number of $C$. difficile-positive specimens was 32 (12.3\%), but in 1 of these a non-toxigenic strain was identified. In addition, two other positive cases detected only by rapid test were toxin-negative. Therefore, the final percentage of toxin-positive cases in the Spanish hospital was established as $11.1 \%$ (29/261). While in the other surveys $[21,23]$ C. difficile was more commonly detected in females, in this study $68.8 \%$ of positive samples were from male patients.

All diarrhoeal non-duplicate specimens submitted to the diagnostic laboratory were tested, even if they were discarded from the routine $C$. difficile detection protocol due to their consistency (samples labelled outside levels 4 to 7 in the BSC). Two positive samples were detected in the analysis of these additional samples ( $\mathrm{n}=12$ ); however, the overall prevalence was almost the same (12.3\% (32/261); $12 \%(30 / 249))$. In a recent study conducted in Australia, while the number of $C$. difficile-positive specimens increased with the analysis of all diarrhoeal specimens (including non-requested samples), the overall prevalence with the analysis of all samples was lower than that identified by routine testing [23]. In a further study conducted in Spain, the authors found that CDI remained a highly neglected disease because of the absence of clinical suspicion or the lack of sensitive diagnostic testing in some 
Table 3

Detailed information on C. difficile-positive patients at the St Luc University Hospital (Belgium), including molecular characterisation of the C. difficile isolates.

\begin{tabular}{|c|c|c|c|c|c|c|c|}
\hline Patient number & Age & Genre & Medical service & C. difficile isolation date & PCR-ribotype & $\mathrm{CE}$ & $t c d A t c d B$ \\
\hline \multirow[t]{4}{*}{01} & 4 years & Male & Paediatric haematology & $01 / 07 / 2014$ & UCL36 & - & - \\
\hline & & & & $04 / 07 / 2014$ & UCL36 & - & - \\
\hline & & & & $08 / 07 / 2014$ & UCL36 & - & - \\
\hline & & & & $17 / 07 / 2014$ & UCL36 & - & - \\
\hline 06 & 28 years & Male & Consultation & $09 / 07 / 2014$ & UCL36 & - & - \\
\hline 11 & 62 years & Male & Medical surgical intensive care & $25 / 08 / 2014$ & UCL36 & - & - \\
\hline 15 & 1 year & Male & Paediatric haematology & $10 / 10 / 2014$ & UCL36 & - & - \\
\hline 56 & 51 years & Female & Gastroenterology & $25 / 08 / 2014$ & UCL36 & - & - \\
\hline \multirow[t]{2}{*}{59} & 81 years & Female & General internal medicine & $02 / 10 / 2014$ & UCL36 & - & - \\
\hline & & & & $09 / 10 / 2014$ & UCL36 & - & - \\
\hline 61 & 88 years & Female & Not specified & $15 / 10 / 2014$ & UCL36 & - & - \\
\hline 63 & 48 years & Female & Consultation & $17 / 10 / 2014$ & UCL36 & - & - \\
\hline 38 & 6 years & Male & Paediatric haematology & $12 / 09 / 2014$ & UCL36a & + & + \\
\hline 82 & 35 years & Female & Urgent care & $01 / 10 / 2014$ & UCL36a & + & + \\
\hline 02 & 4 years & Male & Paediatric haematology & $01 / 07 / 2014$ & UCL9 & - & - \\
\hline 03 & 62 years & Male & Cardiovascular surgery & $07 / 07 / 2014$ & UCL9 & - & - \\
\hline 04 & 64 years & Male & Nephrology neurology & $07 / 07 / 2014$ & UCL9 & - & - \\
\hline 07 & 20 days & Male & Intensive neonatology & $28 / 07 / 2014$ & UCL9 & - & - \\
\hline 08 & 5 months & Male & Outpatient emergency & $04 / 08 / 2014$ & UCL9 & - & - \\
\hline \multirow[t]{3}{*}{12} & 2 years & Male & Paediatric haematology & $26 / 08 / 2014$ & UCL9 & - & - \\
\hline & & & & $01 / 09 / 2014$ & UCL9 & - & - \\
\hline & & & & $22 / 09 / 2014$ & UCL9 & - & - \\
\hline 13 & 10 days & Male & Intensive neonatology & $16 / 09 / 2014$ & UCL9 & - & - \\
\hline 14 & 2 months & Male & Intensive neonatology & $22 / 09 / 2014$ & UCL9 & - & - \\
\hline 19 & 2 years & Male & Not specified & $23 / 10 / 2014$ & UCL9 & - & - \\
\hline 16 & 60 years & Male & Outpatient dialysis & $13 / 10 / 2014$ & UCL9 & - & - \\
\hline 49 & 9 months & Female & Consultation & $01 / 07 / 2014$ & UCL9 & - & - \\
\hline 50 & 14 years & Female & Consultation & $18 / 07 / 2014$ & UCL9 & - & - \\
\hline 53 & 15 days & Female & Intensive neonatology & $28 / 07 / 2014$ & UCL9 & - & - \\
\hline 54 & 84 years & Female & Subacute geriatrics & $04 / 08 / 2014$ & UCL9 & - & - \\
\hline 58 & 8 years & Female & Paediatric haematology & $22 / 09 / 2014$ & UCL9 & - & - \\
\hline 60 & 8 months & Female & Paediatric & $13 / 10 / 2014$ & UCL9 & - & - \\
\hline 05 & 66 years & Male & Pneumatology gastroenterology & $07 / 07 / 2014$ & UCL110 & - & - \\
\hline \multirow[t]{2}{*}{21} & 35 years & Male & Pneumatology gastroenterology & $01 / 07 / 2014$ & UCL100b & + & + \\
\hline & & & & $02 / 07 / 2014$ & UCL100b & + & + \\
\hline 62 & 25 years & Female & Abdominal surgery & $17 / 10 / 2014$ & UCL122 & - & - \\
\hline \multirow[t]{3}{*}{09} & 79 years & Male & Subacute geriatrics & $05 / 08 / 2014$ & UCL257 & - & - \\
\hline & & & Intensive Care Unit & $16 / 10 / 2014$ & UCL257 & - & - \\
\hline & & & Neuro-traumatology & & & & \\
\hline 55 & 66 years & Female & Haematology & $13 / 08 / 2014$ & UCL384 & - & - \\
\hline \multirow[t]{2}{*}{10} & 47 years & Male & Orthopaedics & $19 / 08 / 2014$ & UCL46d & - & - \\
\hline & & & & $25 / 08 / 2014$ & UCL46d & - & - \\
\hline 34 & 94 years & Male & Urgent care & $01 / 09 / 2014$ & UCL48 & + & + \\
\hline 20 & 76 years & Male & General internal medicine & $29 / 10 / 2014$ & UCL122 & - & - \\
\hline 22 & 40 years & Male & General internal medicine & $14 / 07 / 2014$ & UCL 23f & + & + \\
\hline 23 & 46 years & Male & Urgent care & $14 / 07 / 2014$ & UCL86 & + & + \\
\hline 39 & 33 years & Male & Consultation & $16 / 09 / 2014$ & UCL14 & + & + \\
\hline 32 & 82 years & Male & Not specified & $19 / 08 / 2014$ & UCL5a & + & + \\
\hline 86 & 68 years & Female & General internal medicine & $29 / 10 / 2014$ & UCL26 & + & + \\
\hline 72 & 78 years & Female & General internal medicine & $18 / 08 / 2014$ & UCL16r & + & + \\
\hline 44 & 63 years & Male & Not specified & $03 / 10 / 2014$ & UCL16u & + & + \\
\hline 30 & 81 years & Male & General internal medicine & $18 / 08 / 2014$ & UCL16b & + & + \\
\hline 77 & 79 years & Female & Subacute geriatrics & $08 / 09 / 2014$ & UCL16L & + & + \\
\hline 31 & 5 years & Male & Paediatric intensive care & $18 / 08 / 2014$ & UCL16L & + & + \\
\hline \multirow[t]{5}{*}{70} & 58 years & Female & Cardiovascular and thoracic surgery & $13 / 08 / 2014$ & UCL16L & + & + \\
\hline & & & & $29 / 08 / 2014$ & UCL16L & + & + \\
\hline & & & & $01 / 09 / 2014$ & UCL16L & + & + \\
\hline & & & & $01 / 09 / 2014$ & UCL16L & + & + \\
\hline & & & & $07 / 10 / 2014$ & 015 & + & + \\
\hline \multirow[t]{7}{*}{29} & 1 year & Male & Not specified & $11 / 08 / 2014$ & UCL16 ${ }^{\circ}$ & + & + \\
\hline & & & & $12 / 08 / 2014$ & UCL16 $6^{\circ}$ & + & + \\
\hline & & & & $13 / 08 / 2014$ & UCL16 ${ }^{\circ}$ & + & + \\
\hline & & & & $02 / 09 / 2014$ & UCL16 ${ }^{\circ}$ & + & + \\
\hline & & & & $03 / 09 / 2014$ & $\mathrm{UCL}^{\circ} 6^{\circ}$ & + & + \\
\hline & & & & $24 / 09 / 2014$ & $\mathrm{UCL}^{\circ} 6^{\circ}$ & + & + \\
\hline & & & & $27 / 10 / 2014$ & $\mathrm{UCL}^{\circ} 6^{\circ}$ & + & + \\
\hline 57 & 17 years & Female & Paediatric haematology & $30 / 08 / 2014$ & UCL266 & - & - \\
\hline 68 & 36 years & Female & Maternal Intensive Care & $23 / 07 / 2014$ & UCL381 & + & + \\
\hline 52 & 77 years & Female & Nephrology neurology & $22 / 07 / 2014$ & UCL468 & - & - \\
\hline & & & Nephrology neurology & $04 / 08 / 2014$ & UCL468 & - & - \\
\hline & & & Subacute geriatrics & $30 / 08 / 2014$ & 106 & + & + \\
\hline 51 & 34 years & Female & Stomatology neurology & $22 / 07 / 2014$ & UCL471 & - & - \\
\hline 28 & 11 years & Male & Paediatric haematology & $11 / 08 / 2014$ & UCL475 & + & + \\
\hline
\end{tabular}


Table 3 (continued)

\begin{tabular}{|c|c|c|c|c|c|c|c|}
\hline Patient number & Age & Genre & Medical service & C. difficile isolation date & PCR-ribotype & $\mathrm{CE}$ & $\operatorname{tcd} A \operatorname{tcdB}$ \\
\hline 45 & 50 years & Male & Nephrology neurology & $06 / 10 / 2014$ & UCL477 & + & + \\
\hline 17 & 78 years & Male & Subacute geriatrics & $17 / 10 / 2014$ & UCL478 & - & - \\
\hline 18 & 6 months & Male & Ambulatory emergency & $17 / 10 / 2014$ & UCL479 & - & - \\
\hline \multirow[t]{2}{*}{27} & 55 years & Male & Pneumatology Gastroenterology & $31 / 07 / 2014$ & UCL472 & + & + \\
\hline & & & & $07 / 08 / 2014$ & 078 & + & + \\
\hline \multirow[t]{2}{*}{64} & 78 years & Female & Surgery, orthopaedics and traumatology & $02 / 07 / 2014$ & 078 & + & + \\
\hline & & & Subacute geriatrics & $23 / 07 / 2014$ & 078 & + & + \\
\hline \multirow[t]{2}{*}{71} & 64 years & Female & Haematology & $18 / 08 / 2014$ & 078 & + & + \\
\hline & & & & $28 / 08 / 2014$ & 078 & + & + \\
\hline \multirow[t]{2}{*}{40} & 67 years & Male & Pneumatology Gastroenterology & $18 / 09 / 2014$ & 078 & + & + \\
\hline & & & & $18 / 09 / 2014$ & 078 & + & + \\
\hline \multirow[t]{3}{*}{47} & 30 years & Male & Pneumatology Gastroenterology & $17 / 10 / 2014$ & 078 & + & + \\
\hline & & & & $17 / 10 / 2014$ & 078 & + & + \\
\hline & & & & $20 / 10 / 2014$ & 078 & + & + \\
\hline \multirow[t]{2}{*}{75} & 53 years & Female & Oncology & $01 / 09 / 2014$ & 078 & + & + \\
\hline & & & Medical surgical intensive care & $29 / 09 / 2014$ & 078 & + & + \\
\hline 76 & 60 years & Female & Nephrology neurology & $01 / 09 / 2014$ & 078 & + & + \\
\hline \multirow[t]{2}{*}{78} & 57 years & Female & Nephrology neurology & $14 / 10 / 2014$ & 078 & + & + \\
\hline & & & & $23 / 10 / 2014$ & 078 & + & + \\
\hline 80 & 92 years & Female & General internal medicine & $29 / 09 / 2014$ & 078 & + & + \\
\hline 24 & 67 years & Male & Nephrology neurology & $17 / 07 / 2014$ & 014 & + & + \\
\hline \multirow[t]{2}{*}{25} & 90 years & Male & Subacute geriatrics & $30 / 07 / 2014$ & 014 & + & + \\
\hline & & & & $12 / 08 / 2014$ & 014 & + & + \\
\hline 33 & 44 years & Male & Medical surgical intensive care & $27 / 08 / 2014$ & 014 & + & + \\
\hline 81 & 54 years & Female & Gastroenterology & $29 / 09 / 2014$ & 014 & + & + \\
\hline 41 & 29 years & Male & Oncology & $24 / 09 / 2014$ & 020 & + & + \\
\hline 42 & 11 months & Male & Paediatric transplantation & $25 / 09 / 2014$ & 020 & + & + \\
\hline 48 & 1 year & Male & Consultation & $20 / 10 / 2014$ & 020 & + & + \\
\hline 65 & 2 years & Female & Paediatric transplantation & $04 / 07 / 2014$ & 020 & + & + \\
\hline 73 & 1 year & Female & Consultation & $26 / 08 / 2014$ & 020 & + & + \\
\hline \multirow[t]{3}{*}{79} & 68 years & Female & General internal medicine & $09 / 09 / 2014$ & 020 & + & + \\
\hline & & & & $10 / 09 / 2014$ & 020 & + & + \\
\hline & & & & $12 / 09 / 2004$ & 020 & + & + \\
\hline 87 & 7 months & Female & Paediatric transplantation & $30 / 10 / 2014$ & 020 & + & + \\
\hline 35 & 82 years & Male & Pneumatology Gastroenterology & $08 / 09 / 2014$ & 056 & + & + \\
\hline \multirow[t]{2}{*}{83} & 84 years & Female & Not specified & $06 / 10 / 2014$ & 056 & + & + \\
\hline & & & & $14 / 10 / 2014$ & 056 & + & + \\
\hline \multirow[t]{3}{*}{36} & 62 years & Male & Oncology & $09 / 09 / 2014$ & 106 & + & + \\
\hline & & & & $11 / 09 / 2014$ & 106 & + & + \\
\hline & & & & $12 / 09 / 2014$ & 106 & + & + \\
\hline 37 & 71 years & Male & Urgency & $10 / 09 / 2014$ & 106 & + & + \\
\hline 46 & 92 years & Male & Cardiology & $07 / 10 / 2014$ & 106 & + & + \\
\hline 26 & 60 years & Male & Consultation & $30 / 07 / 2014$ & 106 & + & + \\
\hline 84 & 64 years & Female & Nephrology neurology & $20 / 10 / 2014$ & 106 & + & + \\
\hline \multirow[t]{3}{*}{43} & 76 years & Male & Neurology & $01 / 10 / 2014$ & 012 & + & + \\
\hline & & & & $10 / 10 / 2014$ & 012 & + & + \\
\hline & & & & $27 / 10 / 2014$ & 012 & + & + \\
\hline 67 & 85 years & Female & Pneumatology Gastroenterology & $16 / 07 / 2014$ & 012 & + & + \\
\hline 85 & 9 months & Female & Consultation & $24 / 10 / 2014$ & 012 & + & + \\
\hline \multirow[t]{2}{*}{66} & 90 years & Female & Pneumatology Gastroenterology & $09 / 07 / 2014$ & 002 & + & + \\
\hline & & & Cardiology & $05 / 08 / 2014$ & 002 & + & + \\
\hline 69 & 21 years & Female & General internal medicine & $11 / 08 / 2014$ & 002 & + & + \\
\hline 74 & 56 years & Female & Gastroenterology & $01 / 09 / 2014$ & 002 & + & + \\
\hline
\end{tabular}

CE: cytotoxicity assay.

+ : Positive result.

-: Negative result.

institutions. They also observed that underdiagnosis most frequently affected younger patients and patients with communityacquired CDI [22]. In the present study, no positive patients were detected in the paediatric group (less than 10 years old). However, during the study period only 9 samples were received from this service. This data may reflect that in this Spanish hospital a specific request for the diagnosis of $\mathrm{CDI}$ from the clinician is less common in the paediatric service than in others. The mean age of all patients studied (62.5 years old) corroborates this observation. Recent reports warn that the incidence of CDI has increasingly risen among paediatric patients [24]. Collins et al. [23] reported in one survey conducted in Western Australia that undiagnosed CDI cases only occurred among paediatric patients, and $32.3 \%$ of all CDI cases were aged $<20$ years. A further study also conducted in Spain showed that the isolation of $C$. difficile was common in children hospitalised for diarrhoea, especially in patients younger than 2 years old with chronic disease. Furthermore, in the same study the authors reported that the clinical picture observed in children with CDI was characterised by mild symptoms and low clinical severity [25], which may contribute to underdiagnosis in this population. In a previous survey that assessed risk factors and outcomes in children with $C$. difficile-associated diarrhoea, only $12.5 \%$ of positive samples were identified as bloody stools while $79 \%$ of positive samples were watery stools [26]. In a further study conducted in Calcutta to investigate the major clinical features of $C$. difficile-induced diarrhoea, only $17.6 \%$ of C. difficile cases reported bloody stools compared to $84.2 \%$ reporting watery diarrhoea [27]. These reports suggest that bloody stools are not the most common samples 
associated with CDI. Concurrent with these findings, in this study it was observed that all bloody stools tested negative for the bacterium.

In the study of Alcala et al. [22], the second cause proposed for undiagnosed or misdiagnosed CDI was the lack of sensitive diagnostic tests in some institutions. In the present study, four specimens were identified as negative for $C$. difficile and its toxins using rapid tests, but were found to be positive for toxigenic $C$. difficile strains following the culture of samples. In three additional samples toxins were not detected by rapid test but culture, isolation and characterisation of the isolates revealed the presence of at least one of the two toxins A and B. Enzyme immunoassay detection of GDH as initial screening for $C$. difficile presence has been suggested as a potential strategy. However results appear to differ based on the GDH kit used and therefore this approach remains an interim recommendation [2]. While the GenomEra $C$. difficile assay has been described to be an excellent option for toxigenic $C$. difficile detection in faecal specimens [28], in the present study toxigenic $C$. difficile strains were isolated by culture from three samples that were found negative for the toxin using this method. However, the results obtained confirm that GenomEra $C$. difficile assay is more sensitive than EIA testing for $C$. difficile toxin B, as described previously [2]. In contrast, four samples were only positive for $C$. difficile by rapid tests. Two of these positive samples were toxin negative by EIA and Genome $C$. difficile assay. Ethanol shock was not used in the course of this study, nor was alcohol selection of microorganisms conducted, and cultured colonies were observed in high numbers. Therefore, the high contamination of samples by other bacteria species may explain the failure to isolate these four strains by classical culture. For the two non-toxigenic samples identified by rapid screening, false-positive GDH test results are also plausible [29]. The use of the enrichment step in this study was shown to be not useful in the clinical samples tested, as all of the samples that tested positive after 3 enrichment days were already positive by direct culture and the same PCR-ribotypes were isolated.

The surveillance data in Belgium reported a lower prevalence than in Spain (9.3\%). It should be noted that the Spanish and Belgian results must be compared with caution. In the case of the Belgian laboratory, all diarrhoeal faecal specimens were analysed, including duplicate samples from the same patient. In the Spanish hospital only non-duplicate specimens were analysed. Nevertheless, despite this important difference in the routine protocol among laboratories, the prevalence of $C$. difficile is likely to be genuinely lower in Belgium than in Spain. While incidence varies considerably between hospitals and regions, an increase in the proportion of community-associated cases and a decrease in the proportion of hospital-acquired cases of CDI between the years 2008-2014 [30] have been reported in Belgium. This data may reflect the efforts of Belgian hospitals to improve the management and prevention of CDI.

Another important difference found is the mean age of positive patients. In Spain, the mean age found in $C$. difficile-positive cases was 63.6 years old, which correlates with other surveys conducted in the country [22]. In contrast, in Belgium the mean age of adult positive patients was 45 years old. However, if the paediatric group is evaluated separately, the mean age in Belgium is 60 years old. A significant number of positive samples in the Belgian survey were from children less than ten years old $(n=19)$. However, only eight of the positive patients harboured toxigenic $C$. difficile strains. It has been described that during early infancy the gut microbiota complexity is poor and asymptomatic colonisation by $C$. difficile is common [31,32]. However, all the paediatric patient samples analysed in this study were diarrhoeal. These findings corroborate with previous suggestions [23] that the surveillance and the significance of $C$. difficile in paediatric groups requires further investigation.
Toxigenic $C$. difficile carriage in infants could be a cause of disease, not only in paediatric populations but also in adults through close contact with.

In both Spain and Belgium, $C$. difficile-positive patients referred from oncology services all carried toxigenic strains. In a previous study, a great diversity of $C$. difficile strains associated with CDI was detected among paediatric oncology patients [33]. A further study found a probable association between certain types of tumours, the use of antibiotics and CDI incidence. The authors also emphasised the urgent need for early recognition and diagnosis of CDI in adult cancer patients [34]. PCR-ribotypes 078, 014, 012, 020, 002, UCL36a, UCL5a, UCL16b and UCL9 were isolated in both hospitals. In previous surveys in hospitals in Spain, PCR-ribotypes 078/126, 014 and 001 were the most prevalent [22]. As in previous years, PCRribotype 014 remains the most common in Belgium, increasing in proportion to other ribotypes and in the number of hospital sites affected since 2014. The other PCR-ribotypes more commonly detected in Belgian hospitals in 2014 were 020 and 078 [30]. In the Spanish hospital studied, there were no commonly-encountered PCR-ribotypes, suggesting there is neither regional infection nor contamination in the hospital. On the contrary, a great variety of toxigenic PCR-ribotypes was identified. Consistent with the European survey which reported that PCR-ribotype 027 was less prevalent than others [21], this ribotype was not detected either in Spain or Belgium during the present study period.

In conclusion, the data obtained shows that even with three times the number of samples analysed per month, the prevalence of $C$. difficile is lower in the Belgian hospital than the Spanish one. This data may reflect the efforts of the Belgian hospital to improve the management and prevalence of $\mathrm{CDI}$, and, as previously reported, misdiagnosis or underdiagnosis of CDI in Spain due to a lack of clinical suspicion. The most common PCR-ribotypes reported in Europe were found in both hospitals. The great variety of PCRribotypes detected suggests there is neither regional infection nor contamination within the hospital. This study finds that the presence of $C$. difficile in paediatric and oncology services requires further investigation.

\section{Financial support}

This research received no specific grant from any funding agency, commercial or not-for-profit sectors.

\section{Declaration of interest}

None.

\section{Acknowledgements}

The authors offer their most sincere thanks to the microbiology laboratory service of HUCA as well as to the microbiology unit of St Luc Hospital for technical support.

This study was presented at the 5th International Clostridium difficile Symposium (5th ICDS 2014), Bled, Slovenia, May 19-21, 2014.

\section{References}

[1] J.S. Brazier, Clostridium difficile: from obscurity to superbug, Br. J. Biomed. Sci. 65 (1) (2008) 39-44

[2] H. Stuart, et al., Clinical practice guidelines for Clostridium difficile infection in adults: 2010 update by the society for healthcare epidemiology of America (SHEA) and the Infectious Diseases Society of America (IDSA), Infect. Control Hosp. Epidemiol. 31 (5) (2010) 431-455.

[3] M.M. Squire, T.V. Riley, One Health: the Human-animal-environment Interfaces in Emerging Infectious Diseases, vol. 365, Springer Berlin Heidelberg, Berlin, Heidelberg, 2013. 
[4] N.L. Crogan, B.C. Evans, Clostridium difficile: an emerging epidemic in nursing homes, Geriatr. Nurs. (New York, NY) 28 (3) (2007) 161-164.

[5] M. Arvand, et al., High prevalence of Clostridium difficile colonization among nursing home residents in Hesse, Germany, PloS One 7 (1) (2012) e30183.

[6] N. Nanwa, et al., The economic impact of Clostridium difficile infection: a systematic review, Am. J. Gastroenterol. 110 (4) (2015) 511-519.

[7] A.M. Jones, E.J. Kuijper, M.H. Wilcox, Clostridium difficile: a European perspective, J. Infect. 66 (2013) 115-128.

[8] P. Tattevin, et al., Clostridium difficile infections: do we know the real dimensions of the problem? Int. J. Antimicrob. Agents 42 (Suppl. S36-40) (2013).

[9] C.T. Evans, N. Safdar, Current trends in the epidemiology and outcomes of Clostridium difficile infection, Clin. Infect. Dis. Off. Publ. Infect. Dis. Soc. Am. 60 (Suppl. 2) (2015) S66-S71.

[10] L.C. McDonald, et al., An epidemic, toxin gene-variant strain of Clostridium difficile, N. Engl. J. Med. 353 (23) (2005) 2433-2441.

[11] J. Freeman, et al., The changing epidemiology of Clostridium difficile infections, Clin. Microbiol. Rev. 23 (3) (2010) 529-549.

[12] E.J. Kuijper, et al., Update of Clostridium difficile infection due to PCR ribotype 027 in Europe, 2008. Euro Surveillance, Eur. Commun. Dis. Bull. 13 (31) (2008).

[13] G. Van Esch, et al., Surveillance of Clostridium difficile infections in a longterm care psychogeriatric facility: outbreak analysis and policy improvement, Arch. Public Health 73 (1) (2015) 18.

[14] N. Viseur, et al., Nosocomial and non-nosocomial Clostridium difficile infections in hospitalised patients in Belgium: compulsory surveillance data from 2008 to 2010, Euro Surveill. Eur. Commun. Dis. Bull. 16 (43) (2011)

[15] D. Rodríguez-Pardo, et al., Epidemiology of Clostridium difficile infection and risk factors for unfavorable clinical outcomes: results of a hospital-based study in Barcelona, Spain, J. Clin. Microbiol. 51 (5) (2013) 1465-1473.

[16] E. Reigadas, et al., Role of binary toxin in the outcome of Clostridium difficile infection in a non-027 ribotype setting, Epidemiol. Infect. (2015) 1-6.

[17] I. Weber, et al., Molecular epidemiology and resistance profiles of Clostridium difficile in a tertiary care hospital in Spain, Int. J. Med. Microbiol. 303 (3) (2013) 128-133.

[18] C. Rodriguez, et al., Clostridium difficile in young farm animals and slaughter animals in Belgium, Anaerobe 18 (6) (2012) 621-625.

[19] P. Bidet, et al., Development of a new PCR-ribotyping method for Clostridium difficile based on ribosomal RNA gene sequencing, FEMS Microbiol. Lett. 175
(2) (1999) 261-266

[20] Standards - CLSI: CLSI. Available at: http://clsi.org/standards/. (accessed 04.03.15).

[21] M.P. Bauer, et al., Clostridium difficile infection in Europe: a hospital-based survey, Lancet London Engl. 377 (9759) (2011) 63-73.

[22] L. Alcalá, et al., Impact of clinical awareness and diagnostic tests on the underdiagnosis of Clostridium difficile infection, Eur. J. Clin. Microbiol. Infect. Dis. Off. Publ. Eur. Soc. Clin. Microbiol. 34 (8) (2015) 1515-1525.

[23] D.A. Collins, T.V. Riley, Routine detection of Clostridium difficile in Western Australia, Anaerobe 37 (2015) 34-37.

[24] J.S. Sammons, P. Toltzis, Pitfalls in diagnosis of pediatric Clostridium difficile infection, Infect. Dis. Clin. N. Am. 29 (3) (2015) 465-476.

[25] B. Santiago, et al., Clostridium difficile isolation in children hospitalized with diarrhea. Anales de Pediatría (Barcelona, Spain: 2003) 82 (6) (2015) 417-425.

[26] V. Morinville, J. McDonald, Clostridium difficile-associated diarrhea in 200 Canadian children, Can. J. Gastroenterol. 19 (8) (2005) 497-501.

[27] M.K. Bhattacharya, et al., Clinical manifestation of Clostridium difficile enteritis in Calcutta, J. Assoc. Phys. India 39 (9) (1991) 683-684.

[28] J.J. Hirvonen, S. Mentula, S.S. Kaukoranta, Evaluation of a new automated homogeneous PCR assay, GenomEra C. difficile, for rapid detection of Toxigenic Clostridium difficile in fecal specimens, J. Clin. Microbiol. 51 (9) (2013) 2908-2912.

[29] S.D. Goldenberg, P.R. Cliff, G.L. French, Glutamate dehydrogenase for laboratory diagnosis of Clostridium difficile infection, J. Clin. Microbiol. 48 (8) (2010) 3050-3051.

[30] F. Neely and M.L. Lambert. Epidemiology of Clostridium difficile infection in Belgium Report 2015. Available at: http://www.nsih.be/download/CDIF/CDIFAR-2015-EN.pdf. (accessed 27.11.15).

[31] C. Rousseau, et al., Clostridium difficile colonization in early infancy is accompanied by changes in intestinal microbiota composition, J. Clin. Microbiol. 49 (3) (2011) 858-865.

[32] C. Rodriguez, et al., Clostridium difficile infection and intestinal microbiota interactions, Microb. Pathog. 89 (2015) 201-209.

[33] R. Dantes, et al., Investigation of a cluster of Clostridium difficile infections in a pediatric oncology setting, Am. J. Infect. Control 44 (2) (2016) 138-145.

[34] A. Rodríguez Garzotto, et al., Risk factors associated with Clostridium difficile infection in adult oncology patients. Supportive Care in Cancer, Support Care Cancer 23 (6) (2015) 1569-1577. 